\title{
Some completely monotonic functions involving the polygamma functions
}

Peng Gao ${ }^{*}$

"Correspondence:
penggao@buaa.edu.cn
${ }^{1}$ Department of Mathematics,
School of Mathematics and Systems
Science, Beihang University, Beijing,
P.R. China

P.R. China

\begin{abstract}
Motivated by existing results, we present some completely monotonic functions involving the polygamma functions.
\end{abstract}

MSC: 33B15

Keywords: Completely monotonic function; Polygamma functions

\section{Introduction}

The digamma (or psi) function $\psi(x)$ for $x>0$ is defined to be the logarithmic derivative of Euler's gamma function

$$
\Gamma(x)=\int_{0}^{\infty} t^{x} e^{-t} \frac{d t}{t}
$$

The function $\psi$ and its derivatives are called polygamma functions.

There are many interesting inequalities involving the polygamma functions in the literature, many of which are closely related to the fact that $\psi^{\prime}$ is completely monotonic on $(0,+\infty)$. Here we recall that a function $f(x)$ is said to be completely monotonic on $(a, b)$ if it has derivatives of all orders and $(-1)^{k} f^{(k)}(x) \geq 0, x \in(a, b), k \geq 0$, and $f(x)$ is said to be strictly completely monotonic on $(a, b)$ if $(-1)^{k} f^{(k)}(x)>0, x \in(a, b), k \geq 0$.

A general result of Fink [5, Theorem 1] on completely monotonic functions implies that, for integers $n \geq 2$,

$$
\left(\psi^{(n)}(x)\right)^{2} \leq \psi^{(n-1)}(x) \psi^{(n+1)}(x), \quad x>0 .
$$

The following inequality of the reverse direction is given in [11]:

$$
\frac{1}{2} \psi^{\prime}(x) \psi^{\prime \prime \prime}(x) \leq\left(\psi^{\prime \prime}(x)\right)^{2}, \quad x>0 .
$$

A short proof of the above inequality is given in [4].

For integers $p \geq m \geq n \geq q \geq 0$ and any real number $s$, we define

$$
F_{p, m, n, q}(x ; s)=(-1)^{m+n} \psi^{(m)}(x) \psi^{(n)}(x)-s(-1)^{p+q} \psi^{(p)}(x) \psi^{(q)}(x),
$$

where we set $\psi^{(0)}(x)=-1$ for convenience.

(c) The Author(s) 2019. This article is distributed under the terms of the Creative Commons Attribution 4.0 International License (http://creativecommons.org/licenses/by/4.0/), which permits unrestricted use, distribution, and reproduction in any medium, provided you give appropriate credit to the original author(s) and the source, provide a link to the Creative Commons license, and indicate if changes were made. 
In [3, Theorem 2.1], Alzer and Wells established a nice generalization of the above results. Their result asserts that, for $n \geq 2$, the function $F_{n+1, n, n, n-1}(x ; s)$ is strictly completely monotonic on $(0,+\infty)$ if and only if $s \leq(n-1) / n$ and $-F_{n+1, n, n, n-1}(x ; s)$ is strictly completely monotonic on $(0,+\infty)$ if and only if $s \geq n /(n+1)$.

We denote

$$
\begin{aligned}
& \alpha_{p, m, n, q}=\frac{(m-1) !(n-1) !}{(p-1) !(q-1) !}, \quad q \geq 1 ; \\
& \alpha_{p, m, n, 0}=\frac{(m-1) !(n-1) !}{(p-1) !} ; \quad \beta_{p, m, n, q}=\frac{m ! n !}{p ! q !} .
\end{aligned}
$$

Note that $0<\alpha_{p, m, n, q}, \beta_{p, m, n, q}<1$ when $p+q=m+n, p>m$.

In [6, Theorem 4.1], it is shown that, for integers $p>m \geq n>q \geq 0$ satisfying $m+n=$ $p+q$, the function $F_{p, m, n, q}\left(x ; \alpha_{p, m, n, q}\right)$ is completely monotonic on $(0,+\infty)$ and the function $-F_{p, m, n, q}\left(x ; \beta_{p, m, n, q}\right)$ is also completely monotonic on $(0,+\infty)$ when $q>0$. This gives a generalization of the above result of Alzer and Wells.

For a given function $f(x)$, we denote, for $c>0$,

$$
\Delta f(x ; c)=\frac{f(x+c)-f(x)}{c} .
$$

We define, for integers $p \geq m \geq n \geq q \geq 0$, real number $c>0$, and any real number $s$ :

$$
\begin{aligned}
F_{p, m, n, q}(x ; s ; c)= & (-1)^{m+n} \Delta \psi^{(m-1)}(x ; c) \Delta \psi^{(n-1)}(x ; c) \\
& -s(-1)^{p+q} \Delta \psi^{(p-1)}(x ; c) \Delta \psi^{(q-1)}(x ; c),
\end{aligned}
$$

where we set $\psi^{(0)}(x)=\psi(x), \psi^{(-1)}(x)=-x$ for convenience. We further define $F_{p, m, n, q}(x ; s ; 0)=\lim _{c \rightarrow 0^{+}} F_{p, m, n, q}(x ; s ; c)$, and it is then easy to see that $F_{p, m, n, q}(x ; s ; 0)=$ $F_{p, m, n, q}(x ; s)$.

It is shown in [8] that on $(-\min (s, t),+\infty)$ the function $F_{2,1,1,0}(x+s ; 1 ; t-s)$ (resp. its negative) is completely monotonic when $|t-s|<1$ (resp. when $|t-s|<1$ ), and it is further given in [10] a necessary and sufficient condition on $\lambda, t, s$ for $F_{2,1,1,0}(x+s ; \lambda ; t-s)$ or it is negative to be completely monotonic on $(-\min (s, t),+\infty)$. We point out here that one can easily deduce these results on $F_{2,1,1,0}(x+s ; \lambda ; t-s)$ from similar results on $F_{2,1,1,0}(x ; \lambda ; t)$ by a change of variable.

Recently, the complete monotonicity of $F_{p, m, n, q}(x ; s ; c)$ in the case of $(m, n, p, q)=(n, n$, $n-1, n+1$ ) for various $c$ has been determined in [12, Theorem 1]. We also note that the logarithmically complete monotonicity of ratios of two gamma functions is closely related to the divided difference of psi and polygamma functions. There are many known results in this direction, and we refer the reader to recent articles [7, 9, 13-17] and the references therein.

Motivated by the above results, it is our goal in this paper to prove the following:

Theorem 1.1 Let $p>m \geq n>q \geq 0$ be integers satisfying $m+n=p+q$, and let $c>0$. Then

1. For $0<c \leq 1$,

(a) the function $F_{p, m, n, q}(x ; s ; c)$ is completely monotonic on $(0,+\infty)$ if and only if

$$
s \leq \alpha_{p, m, n, q}
$$


(b) the function $-F_{m+n, m, n, 0}(x ; s ; c)$ is completely monotonic on $(0,+\infty)$ if and only if $s \geq \alpha_{m+n, m, n, 0} / c$.

2. For $c \geq 1$,

(a) the function $-F_{p, m, n, q}(x ; s ; c)$ is completely monotonic on $(0,+\infty)$ if and only if $s \geq \alpha_{p, m, n, q}$

(b) the function $F_{m+n, m, n, 0}(x ; s ; c)$ is completely monotonic on $(0,+\infty)$ if and only if $s \leq \alpha_{m+n, m, n, 0} / c$.

3. The function $-F_{p, m, n, q}\left(x ; \beta_{p, m, n, q} ; c\right)$ is completely monotonic on $(0,+\infty)$ for all $c>0$ when $q \geq 1$.

We note here that, when $p=n+1, m=n, q=n-1$, the limiting case $c \rightarrow 0^{+}$of part (1)(a) and part (3) of Theorem 1.1 allows us to recover the above mentioned result of Alzer and Wells. We also point out that by considering finite values of $c$ as done in Theorem 1.1, one is able to see that the monotonicity properties of the function $F_{p, m, n, q}\left(x ; \alpha_{p, m, n, q} ; c\right)$ are different for different values of $c$, while this phenomenon does not occur for the limiting case $c \rightarrow 0^{+}$(as in the result of Alzer and Wells). Another advantage for considering finite values of $c$ instead of the limiting case $c \rightarrow 0^{+}$directly is that one is able to give similar proofs of case 1(a) and (3) for finite values of $c$ in Theorem 1.1. (The proofs corresponding to the limiting case $c \rightarrow 0^{+}$for these two cases in [3] and [6] are different.)

\section{Lemmas}

The first lemma lists some facts about the polygamma functions. These can be found, for example, in [1, (1.1)-(1.3), (1.5)].

Lemma 2.1 For $x>0$,

$$
\begin{aligned}
& \psi(x)=-\gamma+\int_{0}^{\infty} \frac{e^{-t}-e^{-x t}}{1-e^{-t}} d t, \quad \gamma=0.57721 \ldots ; \\
& (-1)^{n+1} \psi^{(n)}(x)=\int_{0}^{\infty} e^{-x t} \frac{t^{n}}{1-e^{-t}} d t, \quad n \geq 1 ; \\
& \psi^{(n)}(x+1)=\psi^{(n)}(x)+(-1)^{n} \frac{n !}{x^{n+1}}, \quad n \geq 0 ; \\
& (-1)^{n+1} \psi^{(n)}(x)=\frac{(n-1) !}{x^{n}}+\frac{n !}{2 x^{n+1}}+O\left(\frac{1}{x^{n+2}}\right), \quad n \geq 1, x \rightarrow+\infty .
\end{aligned}
$$

Lemma 2.2 ([6, Lemma 2.7]) Let $m>n \geq 1$ be two integers, then for any fixed constant $0<c<1$, the function

$$
a(t ; m, n, c)=t^{m-n}+t^{n}-c\left(1+t^{m}\right)
$$

has exactly one root when $t \geq 1$.

Lemma 2.3 Let $a, c>0$, then the function

$$
u(s ; a, c)=\frac{1-e^{-a c(1-s)}}{1-e^{-a(1-s)}} \cdot \frac{1-e^{-a c(1+s)}}{1-e^{-a(1+s)}}
$$

is decreasing on $s \in(0,1)$ if $0<c \leq 1$ and increasing on $(0,1)$ if $c \geq 1$. 
This is Lemma 5 of [2], on setting $r=e^{-a}$ there.

\section{Proof of Theorem 1.1}

We first prove assertions (1)(a) and (2)(a) of the theorem. Note first that if $F_{p, m, n, q}(x ; s ; c)$ is completely monotonic on $(0,+\infty)$, then we have

$$
s \leq \frac{(-1)^{m+n} \Delta \psi^{(m-1)}(x ; c) \Delta \psi^{(n-1)}(x ; c)}{(-1)^{p+q} \Delta \psi^{(p-1)}(x ; c) \Delta \psi^{(q-1)}(x ; c)} .
$$

It then follows easily from the mean value theorem and (2.4) that we have

$$
\lim _{x \rightarrow+\infty} \frac{(-1)^{m+n} \Delta \psi^{(m-1)}(x ; c) \Delta \psi^{(n-1)}(x ; c)}{(-1)^{p+q} \Delta \psi^{(p-1)}(x ; c) \Delta \psi^{(q-1)}(x ; c)}=\alpha_{p, m, n, q}
$$

Thus, $s \leq \alpha_{p, m, n, q}$. Similarly, one shows that if $-F_{p, m, n, q}(x ; s ; c)$ is completely monotonic on $(0,+\infty)$, then $s \geq \alpha_{p, m, n, q}$ and this proves the "only if" part of assertions (1)(a) and (2)(a) of the theorem.

To prove the "if" part of assertions (1)(a) and (2)(a) of the theorem, it is easy to see that it suffices to show that $F_{p, m, n, q}\left(x ; \alpha_{p, m, n, q} ; c\right)$ is completely monotonic on $(0,+\infty)$ when $0<c \leq 1$ and that $-F_{p, m, n, q}\left(x ; \alpha_{p, m, n, q} ; c\right)$ is completely monotonic on $(0,+\infty)$ when $c \geq 1$.

We first consider the function $F_{p, m, n, q}\left(x ; \alpha_{p, m, n, q} ; c\right)$ with $q \geq 1$ following the approach in [3]. Using the integral representations (2.1) and (2.2) for the polygamma functions and using $*$ for the Laplace convolution, we get

$$
F_{p, m, n, q}\left(x ; \alpha_{p, m, n, q} ; c\right)=\int_{0}^{\infty} \frac{e^{-x t}}{c^{2}} g_{p, m, n, q}\left(t ; \alpha_{p, m, n, q}\right) d t,
$$

where

$$
\begin{aligned}
g_{p, m, n, q}\left(t ; \alpha_{p, m, n, q}\right)= & \frac{t^{m-1}\left(e^{-c t}-1\right)}{1-e^{-t}} * \frac{t^{n-1}\left(e^{-c t}-1\right)}{1-e^{-t}} \\
& -\alpha_{p, m, n, q} \frac{t^{p-1}\left(e^{-c t}-1\right)}{1-e^{-t}} * \frac{t^{q-1}\left(e^{-c t}-1\right)}{1-e^{-t}} \\
= & \int_{0}^{t}\left((t-s)^{m-1} s^{n-1}-\alpha_{p, m, n, q}(t-s)^{p-1} s^{q-1}\right) h_{c}(t-s) h_{c}(s) d s,
\end{aligned}
$$

with

$$
h_{c}(s)=\frac{1-e^{-c s}}{1-e^{-s}} .
$$

By a change of variable $s \rightarrow t s$, we can recast $g(t)$ as

$$
\begin{aligned}
& g_{p, m, n, q}\left(t ; \alpha_{p, m, n, q}\right) \\
& \quad=t^{m+n-1} \int_{0}^{1}\left((1-s)^{m-1} s^{n-1}-\alpha_{p, m, n, q}(1-s)^{p-1} s^{q-1}\right) h_{c}(t(1-s)) h_{c}(t s) d s .
\end{aligned}
$$

We now break the above integral into two integrals, one from 0 to $1 / 2$ and the other from $1 / 2$ to 1 . We make a further change of variable $s \rightarrow(1-s) / 2$ for the first one and $s \rightarrow$ 
$(1+s) / 2$ for the second one. We now combine them to get

$$
\begin{aligned}
& g_{p, m, n, q}\left(t ; \alpha_{p, m, n, q}\right) \\
& =\left(\frac{t}{2}\right)^{m+n-1} \int_{0}^{1} a\left(\frac{1+s}{1-s} ; p-q, n-q, \alpha_{p, m, n, q}\right) \\
& \quad \times\left(1-s^{2}\right)^{q-1}(1-s)^{p-q} u(s ; t / 2, c) d s,
\end{aligned}
$$

where the function $a(t ; m, n, c)$ is defined as in Lemma 2.2 and the function $u(s ; a, c)$ is defined as in Lemma 2.3. Note that $(1+s) /(1-s) \geq 1$ for $0 \leq s<1$ and $p-q>n-q \geq 1$, hence by Lemma 2.2 , there is a unique number $0<s_{0}<1$ such that

$$
a\left(\frac{1+s_{0}}{1-s_{0}} ; p-q, n-q, \alpha_{p, m, n, q}\right)=0
$$

It follows from $a\left(1 ; p-q, n-q, \alpha_{p, m, n, q}\right)>0$ and $\lim _{t \rightarrow+\infty} a\left(t ; p-q, n-q, \alpha_{p, m, n, q}\right)<0$ that, for $0<s \leq s_{0}$,

$$
a\left(\frac{1+s}{1-s} ; p-q, n-q, \alpha_{p, m, n, q}\right) \geq 0
$$

with the above inequality being reversed when $s_{0} \leq s<1$.

We further note by Lemma 2.3 that the function $u(s ; t / 2, c)$ is decreasing on $s \in(0,1)$ when $0<c \leq 1$ and increasing when $c \geq 1$. Thus we conclude that, when $0<c \leq 1$,

$$
\begin{aligned}
& a\left(\frac{1+s}{1-s} ; p-q, n-q, \alpha_{p, m, n, q}\right)\left(1-s^{2}\right)^{q-1}(1-s)^{p-q} u(s ; t / 2, c) \\
& \quad \geq a\left(\frac{1+s}{1-s} ; p-q, n-q, \alpha_{p, m, n, q}\right)\left(1-s^{2}\right)^{q-1}(1-s)^{p-q} u\left(s_{0} ; t / 2, c\right),
\end{aligned}
$$

with the above inequality being reversed when $c \geq 1$.

Hence, when $0<c \leq 1$,

$$
\begin{aligned}
& g_{p, m, n, q}\left(t ; \alpha_{p, m, n, q}\right) \\
& \quad \geq\left(\frac{t}{2}\right)^{m+n+1} u\left(s_{0} ; t / 2, c\right) \int_{0}^{1} a\left(\frac{1+s}{1-s} ; p-q, n-q, \alpha_{p, m, n, q}\right)\left(1-s^{2}\right)^{q-1}(1-s)^{p-q} d s,
\end{aligned}
$$

with the above inequality being reversed when $c \geq 1$.

Note that the integral above is (by reversing the process above on changing variables)

$$
2^{m+n-1} \int_{0}^{1}\left((1-s)^{m-1} s^{n-1}-\alpha_{p, m, n, q}(1-s)^{p-1} s^{q-1}\right) d s=0
$$

where the last step follows from the well-known beta function identity

$$
B(x, y)=\int_{0}^{1} t^{x-1}(1-t)^{y-1} d t=\frac{\Gamma(x) \Gamma(y)}{\Gamma(x+y)}, \quad x, y>0
$$


and the well-known fact $\Gamma(n)=(n-1)$ ! for $n \geq 1$. It follows that $g(t) \geq 0$ when $0<c \leq 1$ and $g(t) \leq 0$ when $c \geq 1$, and this completes the proof for the "if" part of assertions (1)(a) and (2)(a) of Theorem 1.1 for $F_{p, m, n, q}\left(x ; \alpha_{p, m, n, q} ; c\right)$ with $q \geq 1$.

Now we consider the function $F_{p, m, n, q}\left(x ; \alpha_{p, m, n, q} ; c\right)$ with $q=0$. In this case $p=m+n$, and we note that

$$
\alpha_{m+n, m, n, 0}=B(m, n)=\int_{0}^{1} s^{m-1}(1-s)^{n-1} d s,
$$

and we use this to write

$$
\alpha_{m+n, m, n, 0} \frac{t^{m+n-1}\left(e^{-c t}-1\right)}{1-e^{-t}}=\int_{0}^{t} \frac{s^{m-1}(t-s)^{n-1}\left(e^{-c t}-1\right)}{1-e^{-t}} d s
$$

It follows that

$$
\begin{aligned}
& F_{m+n, m, n, 0}\left(x ; \alpha_{m+n, m, n, 0} ; c\right) \\
& \quad=\int_{0}^{\infty} \frac{e^{-x t}}{c^{2}}\left(\frac{t^{m-1}\left(e^{-c t}-1\right)}{1-e^{-t}} * \frac{t^{n-1}\left(e^{-c t}-1\right)}{1-e^{-t}}+\alpha_{m+n, m, n, 0} \frac{c t^{m+n-1}\left(e^{-c t}-1\right)}{1-e^{-t}}\right) d t \\
& \quad=\int_{0}^{\infty} \frac{e^{-x t}}{c^{2}}\left(\int_{0}^{t} s^{m-1}(t-s)^{n-1}\left(\frac{1-e^{-c s}}{1-e^{-s}} \cdot \frac{1-e^{-c(t-s)}}{1-e^{-(t-s)}}-\frac{c\left(1-e^{-c t}\right)}{1-e^{-t}}\right) d s\right) d t
\end{aligned}
$$

Now we note that, for $h_{c}(s)$ defined as in (3.1),

$$
\frac{h_{c}^{\prime}(s)}{h_{c}(s)}=-v_{c}(s)
$$

where

$$
v_{c}(x)=\frac{1}{e^{x}-1}-\frac{c}{e^{c x}-1} .
$$

It is easy to see that $v_{c}^{\prime}(x)=z(x, c)-z(x, 1)$ with

$$
z(x, c)=\frac{c^{2} e^{c x}}{\left(e^{c x}-1\right)^{2}} .
$$

Now, we have

$$
\frac{\partial z}{\partial c}=\frac{f(c x) c e^{c x}}{\left(e^{c x}-1\right)^{3}}
$$

where $f(t)=(2-t) e^{t}-(2+t)$. It is then easy to see that $f(t) \leq 0$ for $t \geq 0$, and it follows that $v_{c}^{\prime}(x) \geq 0$ when $0<c \leq 1$ and that $v_{c}^{\prime}(x) \leq 0$ when $c \geq 1$. It follows that $\left(h_{c}^{\prime}(s) / h_{c}(s)\right)^{\prime} \leq 0$ when $0<c \leq 1$ and $\left(h_{c}^{\prime}(s) / h_{c}(s)\right)^{\prime} \geq 0$ when $c \geq 1$. We then deduce that, when $0<c \leq 1$,

$$
\frac{h_{c}^{\prime}(t-s)}{h_{c}(t-s)}-\frac{h_{c}^{\prime}(t)}{h_{c}(t)} \geq 0
$$

for $t \geq s \geq 0$ with the above inequality being reversed when $c \geq 1$. This implies that the function $t \mapsto \ln h_{c}(t-s)-\ln h_{c}(t)$ is increasing (resp. decreasing) for $t>s$ when $0<c \leq 1$ 
(resp. when $c \geq 1$ ). Thus we obtain that, when $0<c \leq 1$,

$$
\ln h_{c}(s)+\ln h_{c}(t-s)-\ln h_{c}(t) \geq \lim _{t \rightarrow s^{+}}\left(\ln h_{c}(s)+\ln h_{c}(t-s)-\ln h_{c}(t)\right)=\ln c,
$$

with the above inequality being reversed when $c \geq 1$. One checks easily that this implies that, when $0<c \leq 1$,

$$
\frac{1-e^{-c(t-s)}}{1-e^{-(t-s)}} \geq \frac{c\left(1-e^{-c t}\right)}{1-e^{-t}}
$$

with the above inequality being reversed when $c \geq 1$. This implies the "if" part of assertions (1)(a) and (2)(a) of the theorem for $F_{m+n, m, n, 0}\left(x ; \alpha_{m+n, m, n, 0} ; c\right)$.

Now we prove assertions (1)(b) and (2)(b) of the theorem. Note that if $F_{m+n, m, n, 0}(x ; s ; c)$ is completely monotonic on $(0,+\infty)$, then we have

$$
s \leq \frac{(-1)^{m+n} \Delta \psi^{(m-1)}(x ; c) \Delta \psi^{(n-1)}(x ; c)}{(-1)^{m+n+1} \Delta \psi^{(m+n-1)}(x ; c)} .
$$

It then follows from (2.3) that we have

$$
\lim _{x \rightarrow 0^{+}} \frac{(-1)^{m+n} \Delta \psi^{(m-1)}(x ; c) \Delta \psi^{(n-1)}(x ; c)}{(-1)^{m+n+1} \Delta \psi^{(m+n-1)}(x ; c)}=\frac{\alpha_{m+n, m, n, 0}}{c} .
$$

Thus, $s \leq \alpha_{m+n, m, n, 0} / c$. Similarly, one shows that if $-F_{m+n, m, n, 0}(x ; s ; c)$ is completely monotonic on $(0,+\infty)$, then $s \geq \alpha_{m+n, m, n, 0} / c$ and this proves the "only if" part of assertions (1)(b) and (2)(b) of the theorem.

To prove the "if" part of assertions (1)(b) and (2)(b) of the theorem, it is easy to see that it suffices to show that $-F_{m+n, m, n, 0}\left(x ; \alpha_{m+n, m, n, 0} / c ; c\right)$ is completely monotonic on $(0,+\infty)$ when $0<c \leq 1$ and that $F_{m+n, m, n, 0}\left(x ; \alpha_{m+n, m, n, 0} / c ; c\right)$ is completely monotonic on $(0,+\infty)$ when $c \geq 1$.

Similarly to (3.3), we have

$$
\begin{aligned}
& F_{m+n, m, n, 0}\left(x ; \frac{\alpha_{m+n, m, n, 0}}{c} ; c\right) \\
& \quad=\int_{0}^{\infty} \frac{e^{-x t}}{c^{2}}\left(\int_{0}^{t} s^{m-1}(t-s)^{n-1}\left(\frac{1-e^{-c s}}{1-e^{-s}} \cdot \frac{1-e^{-c(t-s)}}{1-e^{-(t-s)}}-\frac{\left(1-e^{-c t}\right)}{1-e^{-t}}\right) d s\right) d t .
\end{aligned}
$$

For fixed $t>s>0$, define

$$
r_{s, t}(c)=\frac{\left(1-e^{-c s}\right)\left(1-e^{-c(t-s)}\right)}{1-e^{-c t}} .
$$

Then we have

$$
c \frac{r_{s, t}^{\prime}(c)}{r_{s, t}(c)}=\frac{c s}{e^{c s}-1}+\frac{c(t-s)}{e^{c(t-s)}-1}-\frac{c t}{e^{c t}-1}>0,
$$

as it is easy to see that the function $x \mapsto x /\left(e^{x}-1\right)$ is decreasing for $x>0$. It follows that the function $r_{s, t}(c)$ is an increasing function of $c$ so that $r_{s, t}(c) \leq r_{s, t}(1)$ when $0<c \leq 1$ and 
$r_{s, t}(c) \geq r_{s, t}(1)$ when $c \geq 1$. One sees easily that the "if" part of assertions (1)(b) and (2)(b) of the theorem follows from this.

Lastly, we prove assertion (3) of the theorem. This is similar to our proof above of the "if" part of assertions (1)(a) and (2)(a) of the theorem for $F_{p, m, n, q}\left(x ; \alpha_{p, m, n, q} ; c\right)$ with $q \geq 1$, except that we replace $\alpha_{p, m, n, q}$ by $\beta_{p, m, n, q}$ and recast the function $g_{p, m, n, q}\left(t ; \beta_{p, m, n, q}\right)$ similar to $(3.2)$ as

$$
\begin{aligned}
& g_{p, m, n, q}\left(t ; \beta_{p, m, n, q}\right) \\
& =\left(\frac{t}{2}\right)^{m+n+1} \int_{0}^{1} a\left(\frac{1+s}{1-s} ; p-q, n-q, \beta_{p, m, n, q}\right) \\
& \quad \times\left(1-s^{2}\right)^{q}(1-s)^{p-q}\left(\frac{t^{2}}{4}\left(1-s^{2}\right)\right)^{-1} u(s ; t / 2, c) d s .
\end{aligned}
$$

It is then easy to show using the method in the proof of Lemma 2.3 that the function $s \mapsto a^{-2}\left(1-s^{2}\right)^{-1} u(s ; a, c)$ is increasing on $s \in(0,1)$ when $c>0$, and essentially repeating the rest of the proof of the "if" part of assertions (1)(a) and (2)(a) of the theorem allows us to establish assertion (3) of the theorem.

\section{Acknowledgements}

The author is grateful to the referees for many helpful suggestions.

Funding

Not applicable.

Availability of data and materials

Data sharing not applicable to this article as no datasets were generated or analysed during the current study.

Competing interests

The author declares that there are no competing interests.

Authors' contributions

The author completed the paper and approved the final manuscript.

\section{Publisher's Note}

Springer Nature remains neutral with regard to jurisdictional claims in published maps and institutional affiliations.

Received: 25 March 2019 Accepted: 7 August 2019 Published online: 20 August 2019

\section{References}

1. Alzer, H.: Sharp inequalities for the digamma and polygamma functions. Forum Math. 16, 181-221 (2004)

2. Alzer, H.: Sub- and superadditive properties of Euler's gamma function. Proc. Am. Math. Soc. 135, 3641-3648 (2007)

3. Alzer, H., Wells, J.: Inequalities for the polygamma functions. SIAM J. Math. Anal. 29, 1459-1466 (1998)

4. English, B.J., Rousseau, G.: Bounds for certain harmonic sums. J. Math. Anal. Appl. 206, 428-441 (1997)

5. Fink, A.M.: Kolmogorov-Landau inequalities for monotone functions. J. Math. Anal. Appl. 90, 251-258 (1982)

6. Gao, P.: Some monotonicity properties of gamma and q-gamma functions. ISRN Math. Anal. 2011, Article ID 375715 (2011)

7. Qi, F., Agarwal, R.P.: On complete monotonicity for several classes of functions related to ratios of gamma functions. J. Inequal. Appl. 2019, 36 (2019)

8. Qi, F., Guo, B.-N.: Completely monotonic functions involving divided differences of the di- and tri-gamma functions and some applications. Commun. Pure Appl. Anal. 8, 1975-1989 (2009)

9. Qi, F., Guo, B.-N.: Complete monotonicity of divided differences of the di- and tri-gamma functions with applications. Georgian Math. J. 23, 279-291 (2016)

10. Qi, F., Guo, B.-N.: Necessary and sufficient conditions for a function involving divided differences of the di- and tri-gamma functions to be completely monotonic. arXiv:0903.3071

11. Trimble, S.Y., Wells, J., Wright, F.T.: Superadditive functions and a statistical application. SIAM J. Math. Anal. 20, 1255-1259 (1989)

12. Yang, Z.-H.: Some properties of the divided difference of psi and polygamma functions. J. Math. Anal. Appl. 455 761-771 (2017)

13. Yang, Z.-H., Tian, J.-F.: A comparison theorem for two divided differences and applications to special functions. J. Math. Anal. Appl. 464, 580-595 (2018) 
14. Yang, Z.-H., Tian, J.-F.: A class of completely mixed monotonic functions involving the gamma function with applications. Proc. Am. Math. Soc. 146, 4707-4721 (2018)

15. Yang, Z.-H., Tian, J.-F.: Monotonicity rules for the ratio of two Laplace transforms with applications. J. Math. Anal. Appl. 470, 821-845 (2019)

16. Yang, Z.-H., Zheng, S.-Z.: Complete monotonicity involving some ratios of gamma functions. J. Inequal. Appl. 2017, $255(2017)$

17. Yang, Z.-H., Zheng, S.-Z.: Complete monotonicity and inequalities involving Gurland's ratios of gamma functions. Math. Inequal. Appl. 22, 97-109 (2019)

Submit your manuscript to a SpringerOpen ${ }^{\circ}$ journal and benefit from:

- Convenient online submission

- Rigorous peer review

- Open access: articles freely available online

- High visibility within the field

- Retaining the copyright to your article

Submit your next manuscript at $\gg$ springeropen.com 